\title{
The Effect of Lower Extremity Strengthening Exercise Using Sliding Stander on Balance and Spasticity in Chronic Stroke: A Randomized Clinical Trial
}

\author{
Byeong Mu Mun', Jin Park², Tae Ho Kim² \\ ${ }^{1}$ Kumi Movement Development Center, Jeonju; ${ }^{2}$ Department of Physical Therapy, Graduate School, Daegu University, Daegu, Korea
}

\begin{abstract}
Purpose: Generally, patients with stroke present with decreased balance and increased spasticity following weakness of the paralyzed muscles. Muscle weakness caused by stroke has two causes. This is caused by a decrease in motor output and an adaptive muscle change, resulting in muscle weakness and muscle paralysis. The purpose of this study was to investigate the effect of strengthening exercise on balance and spasticity in chronic stroke patients and to suggest the basis of clinical treatment.

Methods: Twenty subjects were divided into two groups: a lower-extremity strengthening group (experimental group) and a general physical therapy group (control group). The sliding stander equipment was used for the experimental group and a regimen of warm-up exercise, the main exercise routine, and cool-down exercise were used for the muscle strengthening exercise program. Balance and spasticity were measured before and after the training period. Balance ability was measured by the Berg balance scale, the Timed up and Go test and the weight distribution of the paralyzed muscles by the Spacebalance 3D. Spasticity was measured by the Biodex system.

Results: After the training periods, the experimental group showed a significant improvement in BBS, weight distribution of the paralyzed muscles, and decreased spasticity when compared to the control group $(p<0.05)$.

Conclusion: This study supported the hypothesis that lower-extremity strengthening exercise improves the balance and decreases the spasticity of stroke patients. If it is combined with conventional neurologic physiotherapy, it would be effective rehabilitation for stroke patients.
\end{abstract}

Keywords: Balance, Spasticity, Strengthening, Stroke

\section{INTRODUCTION}

Stroke patients experience balance and walking disorders due to multiple functional disorders, indicating limitations of functional activities required for independent daily living. ${ }^{1}$ In particular, muscle weakness is an element limiting the functional rehabilitation of stroke patients and affects balance and walking ability, thus becoming a treatment goal of assessing functional ability. ${ }^{2}$ Muscle weakness caused by stroke has two causes. The first cause is a reduction in the number of motor units that can be mobilized as a result of a decrease in motor output. The second cause is both a lack of muscle activity and lack of exercise. ${ }^{3}$ This is caused by a decrease in motor output and an adaptive muscle change, resulting in muscle weak-

Received Sep 11, 2019 Revised Sep 29, 2019

Accepted Sep 30, 2019

Corresponding author Tae Ho Kim

E-mail hohoho90@naver.com ness and muscle paralysis. ${ }^{4}$

In general, hemiplegia patients with stroke lose weight on the lower extremities when standing due to musculoskeletal problems, such as sensory impairment and muscle weakness. This results in a asymmetric position characteristics. ${ }^{5}$ In addition, patients with stroke have over-used the paralyzed side for an extended period. This results in weakness of the upper and lower limbs on the paralyzed side. ${ }^{6}$ Abnormal muscle recruitment also reduces the endurance required for weight-bearing and during activity, as the center of pressure is concentrated on the less paralyzed side and the postural sway increases?

Spasticity has positive aspects, such as stability in standing or walking, maintaining muscle mass, and maintaining bone density. ${ }^{8}$

Copylight (C)2019 The Korean Society of Physical Therapy

This is an Open Access article distribute under the terms of the Creative Commons Attribution Non-commercial License (Http:// creativecommons.org/license/by-nc/4.o.) which permits unrestricted non-commercial use, distribution, and reproduction in any medium, provided the original work is properly cited. 
On the other hand, there is a difficulty in maintaining balance due to interfering with the motor function by increasing the synergy pattern, resulting in negative outcomes such as joint contracture, pain, and muscle spasms. ${ }^{8,9}$ Therefore, a treatment that minimizes the adverse effects of spasticity after brain lesions is an important goal in clinical practice. ${ }^{10}$

To minimize the negative effects of balance disorders and spasticity in patients with stroke, clinical strength training was conducted at the clinic. Progressive resistance exercise of the lower limbs conducted through the application of functional performance in stroke patients has had significant effects on lower-limb strength, balance, and walking ability. ${ }^{11,12}$ Byun et al. ${ }^{13}$ reported that after applying the sliding rehabilitation device for the treatment of stroke patients with hemiplegia, improved strength, stiffness, walking ability, balance, and ability to perform daily activities resulted. In patients with stroke, progressive resistance exercise $\mathrm{e}^{14}$ and isokinetic muscle strengthening exercises ${ }^{15}$ increased muscle strength without increasing stiffness and contraction. Repeated muscle strengthening exercise has been shown to maintain muscle length and effectively reduce spasticity.

However, most of the previous studies on the effects of muscle strengthening exercise have not focused on spasticity and balancerelated issues that negatively affect the daily life activities of stroke patients. In addition, even in studies related to spasticity, there was no quantitative measurement of spasticity measured using the modified Ashworth scale. The purpose of this study was to investigate the effect of strengthening exercise on balance and spasticity in chronic stroke patients and to suggest the basis for clinical treatment.

\section{METHODS}

\section{Subjects}

The research subjects were 20 patients diagnosed with strokes at the hospital in K hospital in Jeonju of Korea. The subjects sufficiently understood the study procedure and submitted informed written consent to participate in this study. The study was conducted in accordance with the Declaration of Helsinki.

The inclusion criteria for the subjects who had a stroke were (1) more than 6 months after the onset of stroke and less than 2 years, (2) the ability to walk for 10 minutes or longer on a treadmill, (3) absence of neurotic diseases such as amblyopia, vertigo, and abnormal vestibular function, (4) Subjects had no orthopedic problems that
Table 1. General characteristics of subjects

\begin{tabular}{lcc}
\hline & $\begin{array}{c}\text { Experimental group } \\
(\mathrm{n}=10)\end{array}$ & $\begin{array}{c}\text { Control group } \\
(\mathrm{n}=10)\end{array}$ \\
\hline Age (yr) & $53.1 \pm 13.4$ & $54.0 \pm 9.1$ \\
Gender (Male/Female) & $8 / 2$ & $8 / 2$ \\
Time since stroke (Month) & $20.3 \pm 14.4$ & $15.8 \pm 10.2$ \\
Type of lesion (\%) & & \\
Hemorrhagic & $7(70)$ & $3(30)$ \\
Infarction & $3(30)$ & $7(70)$ \\
Side of lesion (Rt/Lt) & $3 / 7$ & $5 / 5$ \\
Height (cm) & $167 \pm 8.2$ & $164.4 \pm 9$ \\
Weight (kg) & $65.6 \pm 7.4$ & $66.1 \pm 6.9$ \\
MAS (Score) & $2.2 \pm 0.6$ & $2.2 \pm 0.7$ \\
\hline
\end{tabular}

Mean \pm standard deviation. MAS: Modified ashworth scale.

could affect exercise in the lower limbs, (5) cognitive function allowing an understanding of researchers' instructions. Table 1 summarizes the subjects general characteristics (Table 1).

\section{Experimental methods}

\section{1) Experimental procedures}

Subjects who participated in this study were randomly assigned to two groups, which paired subjects with similar physical abilities. Lower-extremity strengthening (experimental) group consisted of 10 people, and the general physical therapy (control) group consisted of 10 people. Both the experimental group and the control group received neurodevelopment therapy. However, the experimental group performed additional lower-extremity strengthening training for 30 minutes, five times a week, for six weeks.

The experimental group performed lower-extremity strength training on the MET 300 (Met 300, CyberMedic Co., Ltd., Iksan, Korea). The subjects bent their knees 90 degrees in a comfortable position on the backrest mat, supported the footrest, and bent the elbows of the two arms in a lying position to fix the hands to the chest. After observing the frontal positions, the upper body was kept intact, and the knee flexion and extension were repeated. The warmup exercise and cool-down exercise were performed on the MET 300 with knee flexion and extension repeated for five minutes with $25 \%$ of 1 RM force. In the main exercise, based on Holten's study, we performed a total of three sets of 15 to 20 repeated for twenty minutes with $70 \%$ power of 1 RM. ${ }^{12}$ The MET 300 angle was adjusted to maintain $70 \%$ of 1 RM with increasing muscle strength (Figure 1). In the event that a subject complained of fatigue during training, a break was provided to minimize his/her fatigue level. In addition, 

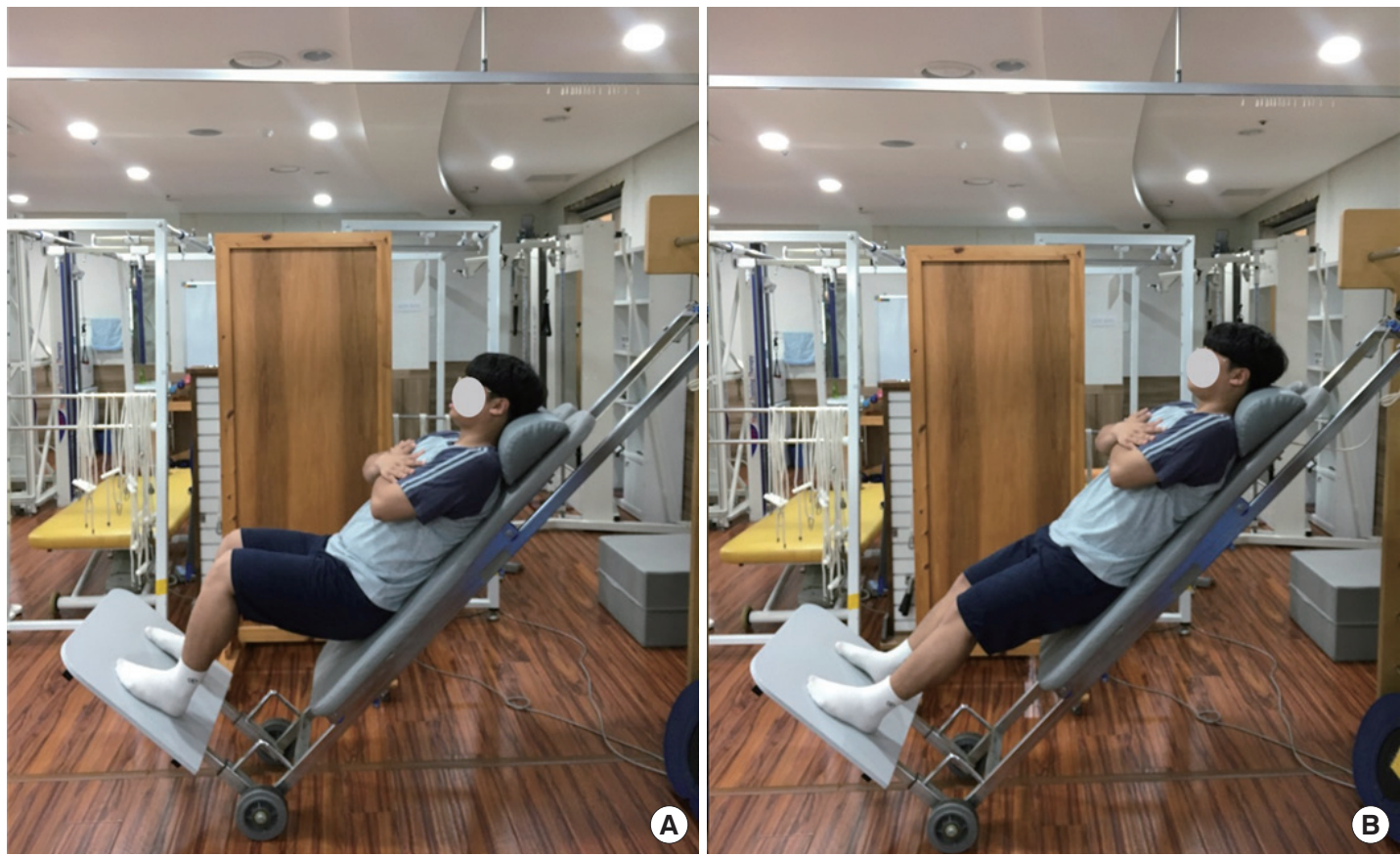

Figure 1. Lower-extremity strengthening exercise using sliding stander. (A) Start position, (B) End position.

one physical therapist assisted each subject to safety.

The control group corrected the abnormal postures and movements as well as promoted the lower-extremity normal exercise pattern and joint movement exercise, balance training, and gait training, respectively. If a subject complained of fatigue, a break was given to minimize his/her fatigue level, and physical therapist assisted each subject to safety.

\section{2) Assessment}

The Berg balance scale (BBS) and timed up and go (TUG) tests were used to measure functional balance ability. In order to measure the static balance ability of all subjects, the weight-bearing distribution of the paretic side was measured by subjects maintaining a standing position on a Spacebalance 3D (Spacebalance 3D, CyberMedic Co., Ltd., Iksan, Korea). This study evaluated the weight-bearing distribution on the paretic side during static standing with eyes open and closed to confirm the weight-bearing distribution of the standing posture.

The Biodex system (Biodex Medical System Inc., NY, USA) was used to measure spasticity. The subject is allowed to sit on a Biodex system chair and the knee joint of the lower leg is fixed at $30^{\circ} \mathrm{flex}-$ ion. The axis of the ankle joint were aligned with the axis of the Biodex system. The isokinetic manual extension exercise was performed at an angular velocity of $60 \% \mathrm{sec}, 180 \% \mathrm{sec}$, and $240 \% \mathrm{sec}$, and five times per velocity at a joint angle range of $30^{\circ}$ to an ankle plantar flexion and $20^{\circ}$ of an ankle dorsi flexion. Eccentric torques were measured and had a five-second rest period at each speed between manual extension exercise. ${ }^{16}$

\section{Statistical analysis}

PASW 18.0 version for Window (PASW Inc., Chicago, IL, USA) was used for statistical analysis of the measured values of the subjects. Descriptive statistics were used to present each variable as mean \pm standard deviation. The Kolmogorov-Smirnov test was used to test the normality. To examine the differences in general characteristics between the two groups, the independent t-test were used. To verify the differences within each group between pre-test and post-test balance ability, spasticity the paired t-test was used, and the independent t-test was used to verify the differences between the two groups. The significance level was set to 0.05 .

\section{RESULTS}

\section{Functional balance ability}

Among the functional balance ability, the results of a comparison before and after the training showed that both group's BBS statistically significantly increased $(\mathrm{p}<0.05)$. After the training, the results 
Table 2. Berg balance scale (BBS) and Timed up and go (TUG) test results for the experimental and control groups

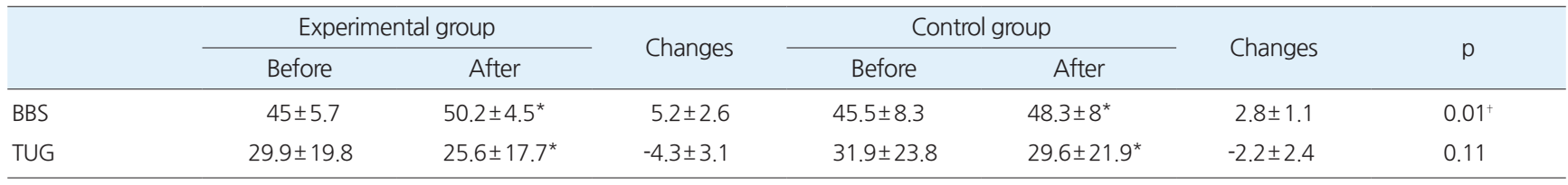

Mean \pm standard deviation. Significant difference between pre and post intervention within the group $\left({ }^{*} \mathrm{p}<0.05\right)$, significant difference between the change values among the groups $\left({ }^{+} p<0.05\right)$.

Table 3. Affected weight distribution results for the experimental and control groups

\begin{tabular}{|c|c|c|c|c|c|c|c|}
\hline & \multicolumn{2}{|c|}{ Experimental group } & \multirow{2}{*}{ Changes } & \multicolumn{2}{|c|}{ Control group } & \multirow{2}{*}{ Changes } & \multirow{2}{*}{$\mathrm{p}$} \\
\hline & Before & After & & Before & After & & \\
\hline Affected weight distribution (open eyes) & $44.9 \pm 3.4$ & $48 \pm 2.2^{*}$ & $3 \pm 2.2$ & $45.5 \pm 3.1$ & $46.7 \pm 3.4^{*}$ & $1.2 \pm 1.3$ & $0.04^{+}$ \\
\hline Affected weight distribution (closed eyes) & $43.8 \pm 2.2$ & $48 \pm 2.4^{*}$ & $4.2 \pm 2.3$ & $43.8 \pm 2.3$ & $46.1 \pm 2.3^{*}$ & $2.3 \pm 1.2$ & $0.03^{+}$ \\
\hline
\end{tabular}

Mean \pm standard deviation. Significant difference between pre and post intervention within the group $\left({ }^{*} \mathrm{p}\langle 0.05)\right.$, significant difference between the change values among the groups $\left({ }^{+} \mathrm{p}<0.05\right)$.

Table 4. Spasticity results for the experimental and control groups

\begin{tabular}{|c|c|c|c|c|c|c|c|}
\hline & \multicolumn{2}{|c|}{ Experimental group } & \multirow{2}{*}{ Changes } & \multicolumn{2}{|c|}{ Control group } & \multirow{2}{*}{ Changes } & \multirow{2}{*}{$p$} \\
\hline & Before & After & & Before & After & & \\
\hline $60 \% / \sec (\%)$ & $39.2 \pm 14.4$ & $31.6 \pm 12^{*}$ & $7.7 \pm 6.6$ & $27.5 \pm 9.5$ & $24.5 \pm 7.8$ & $3 \pm 4.2$ & 0.07 \\
\hline $180^{\circ} / \mathrm{sec}(\%)$ & $43.7 \pm 14.5$ & $33.1 \pm 10.8^{*}$ & $10.5 \pm 8.4$ & $31.4 \pm 10.9$ & $28.5 \pm 9.4^{*}$ & $2.9 \pm 3.6$ & $0.02^{+}$ \\
\hline $240^{\circ} / \mathrm{sec}(\%)$ & $42.9 \pm 14.2$ & $32.5 \pm 9.1^{*}$ & $10.4 \pm 8.4$ & $31.4 \pm 10.9$ & $27.8 \pm 8.9^{*}$ & $3.7 \pm 4.4$ & $0.04^{+}$ \\
\hline
\end{tabular}

Mean \pm standard deviation. Significant difference between pre and post intervention within the group $\left({ }^{*} p<0.05\right)$, significant difference between the change values among the groups $(+p<0.05)$

of a comparison between the two groups showed that the experimental group's BBS statistically significantly increased compared to the control group $(\mathrm{p}<0.05)$.

The results of a comparison before and after the training showed that both group's TUG statistically significantly decreased $(\mathrm{p}<0.05)$. After the training, no statistically significant differences were observed between the two groups $(\mathrm{p}<0.05)$ (Table 2).

\section{Static balance ability}

The results of a comparison before and after the training showed that both group's weight distribution of the paralyzed at the eyes open and the eyes closed statistically significantly increased $(\mathrm{p}<0.05)$. After the training, the results of a comparison between the two groups showed that the experimental group's weight distribution of the paralyzed statistically significantly increased compared to the control group $(\mathrm{p}<0.05)$ (Table 3$)$.

\section{Spasticity}

The results of a comparison before and after the training showed that the experimental group's spasticity statistically significantly de- creased at an angular velocity of $60 \% \mathrm{sec}, 180^{\circ} / \mathrm{sec}$, and $240 \% \mathrm{sec}$ $(\mathrm{p}<0.05)$. The results of a comparison before and after the training showed that the control group's spasticity statistically significantly decreased at an angular velocity of $180^{\circ} / \mathrm{sec}$, and $240 \% \mathrm{sec}(\mathrm{p}<0.05)$. After the training, the results of a comparison between the two groups showed that the experimental group's spasticity statistically significantly decreased compared to the control group at an angular velocity of $180 \% \mathrm{sec}$, and $240 \% \mathrm{sec}(\mathrm{p}<0.05)$ (Table 4$)$.

\section{DISCUSSION}

This study intended to suggest an effective clinical method of training stroke patients to improve their balance and decrease their spasticity with and without lower-extremity strengthening.

As a result, BBS and TUG, which were measured to evaluate functional balance ability showed a statistically significant improvement after the intervention. After the training, a comparison between the two groups showed that the experimental group's BBS showed a statistically significant increase as compared to the control group. Similar to the results of this study, Tung et al. ${ }^{17}$ reported that strengthen- 
ing exercise through sit-to-standing training was effective in improving the balance of stroke patients. On the other hand, Mercer et al. ${ }^{18}$ reported that when the hip abductor strengthening exercise was applied to stroke patients, paralyzed and non-paralyzed muscles improved, but no significant change in BBS score was observed. This is due to muscle strengthening exercise that was limited to only the hip abductors. In order to maintain balance, muscle strength is required not only in the hip joint but also in multiple joints. ${ }^{19}$ Both a previous study and this study reported that muscle strengthening exercise is effective in improving balance ability. It is believed that BBS and TUG have improved results because they are various muscle movements of the muscles related to both the knee joint and ankle joint, and not focusing on one joint muscle.

In the static balance ability evaluation, based on the weight distribution of the paralyzed subjects at the time of standing, the results from static standing with both the eyes-open and eyes-closed showed statistically significant improvement compared with those before the intervention. After the training, the results of a comparison between the two groups showed that the experimental group's weight distribution showed a statistically significant increase compared to the control group. Similarly, muscle strengthening exercise through sitto-standing training for stroke patients has been reported to improve the weight distribution on the paralyzed side., ${ }^{2,17}$ Muscle strength associated with the knee joint and ankle joint is important in performing sit-to-standing exercise. ${ }^{20}$ The weight distribution on the paralyzed side is improved due to increased muscle strength related to the paralyzed knee joint and ankle joint through sit-to-standing training. However, performing sit-to-standing training can be challenging for stroke patients. Therefore, it is necessary to perform strength training in a stable state. In this study, the muscle strength of the knee joint and ankle joint muscles was improved and the weight distribution of the paralyzed side was changed by the muscle-strengthening exercise performed with the MET instrument.

Spasticity was statistically significantly decreased after intervention applied to both groups at an angular velocity of $180 \% \mathrm{sec}$ and $240 \% \mathrm{sec}$. After the training, the results of a comparison between the two groups showed that the experimental group's spasticity showed a statistically significant decrease compared to the control group at an angular velocity of $180 \% \mathrm{sec}$, and $240 \% \mathrm{sec}$. This means that lower-extremity strengthening exercise is effective in reducing spasticity. Engardt et al. ${ }^{21}$ argued that the spasticity of a stroke patient was exacerbated by muscle weakness and progressive strengthening exercise can lead to a reduction in spasticity due to the maintenance of muscle length. In this study, we also confirmed that spasticity reduction was achieved through lower-extremity strengthening exercises, resulting from the repetition contraction and elongation caused by the muscle-strengthening exercise; this improved neurological control and maintained the length of the muscles. In addition, compared with the control group, the decrease in spasticity in the experimental group was thought to be due to the repeated strengthening exercise reduction of the knee joint and ankle joint muscles through MET 300.

Methods for assessing spasticity in clinical settings include a subjective method of assessing the degree of resistance when the joint is manually moved, such as the modified Ashworth scale, ${ }^{22}$ the tendon reflex test using the spinal cord reflex, and an indirect method using ground reaction force during walking. ${ }^{23}$ There is also a method using isokinetic muscle strength. ${ }^{16}$ Eccentric torques measured by the isokinetic muscle strength were a clinical indicator of spasticity and significantly correlated with the modified Ashworth scale, which is an index reflecting the degree of spasticity. ${ }^{16}$ The isokinetic manual extension exercise was performed at an angular velocity of $60 \% \mathrm{sec}$, $180^{\circ} / \mathrm{sec}$, and $240^{\circ} / \mathrm{sec}$, at a joint angle range of $30^{\circ}$ to an ankle plantar flexion and $20^{\circ}$ of an ankle dorsi flexion and measured eccentric torques. It is possible to objectively evaluate the spasticity of patients with stroke objectively, but other methods should be considered, as this method is limited to objectively evaluating the spasticity in the absence of isokinetic muscle strength in clinical practice.

This study had certain limitations. The number of subjects was small, and there was a marked difference in functional levels among the subjects, which is selecting the subjects, considered only the duration of the disease and the degree of spasticity. In addition, no confirmation was made whether the effect of treatment remained constant after the intervention. Long-term studies with more subjects will be needed for future studies to determine how well various enhancement methods for stroke patients can provide more effective rehabilitation.

\section{REFERENCES}

1. Geurts AC, de Haart M, van Nes IJ et al. A review of standing balance recovery from stroke. Gait Posture. 2005;22(3):267-81.

2. Bohannon RW. Muscle strength and muscle training after stroke. J Rehabil Med. 2007;39(1):14-20. 
3. Farmer SF, Swash M, Ingram DA et al. Changes in motor unit synchronization following central nervous lesions in man. J Physiol. 1993; 463(1):83-105.

4. Gemperline JJ, Allen S, Walk D et al. Characteristics of motor unit discharge in subjects with hemiparesis. Muscle Nerve. 1995;18(10):110114.

5. Eng JJ, Chu KS. Reliability and comparison of weight-bearing ability during standing tasks for individuals with chronic stroke. Arch Phys Med Rehabil. 2002;83(8):1138-44.

6. Campbell FM, Ashburn AM, Pickering RM et al. Head and pelvic movements during a dynamic reaching task in sitting: implications for physical therapists. Arch Phys Med Rehabil. 2001;82(12):1655-60.

7. Laufer Y, Dickstein R, Resnik S et al. Weight-bearing shifts of hemiparetic and healthy adults upon stepping on stairs of various heights. Clin Rehabil. 2000;14(2):125-9.

8. Rekand T. Clinical assessment and management of spasticity: a review. Acta Neurol Scand Suppl. 2010;(190):62-6.

9. O'Dell MW, Lin CC, Harrison V. Stroke rehabilitation: strategies to enhance motor recovery. Annu Rev Med. 2009;60:55-68.

10. Francisco GE, McGuire JR. Poststroke spasticity management. Stroke. 2012;43(11):3132-6.

11. Bale M, Strand LI. Does functional strength training of the leg in subacute stroke improve physical performance? A pilot randomized controlled trial. Clin Rehabil. 2008;22(10-11):911-21.

12. Ouellette MM, LeBrasseur NK, Bean JF et al. High-intensity resistance training improves muscle strength, self-reported function, and disability in long-term stroke survivors. Stroke. 2004;35(6):1404-9.

13. Byun SD, Jung TD, Kim CH et al. Effects of the sliding rehabilitation machine on balance and gait in chronic stroke patients - a controlled clinical trial. Clin Rehabil. 2011;25(5):408-15.
14. Miller GJ, Light KE. Strength training in spastic hemiparesis: should it be avoided? NeuroRehabilitation. 1997;9(1):17-28.

15. Sharp SA, Brouwer BJ. Isokinetic strength training of the hemiparetic knee: effects on function and spasticity. Arch Phys Med Rehabil. 1997; 78(11):1231-6.

16. Freire B, Dias CP, Goulart NB et al. Achilles tendon morphology, plantar flexors torque and passive ankle stiffness in spastic hemiparetic stroke survivors. Clin Biomech. 2017;41:72-6.

17. Tung FL, Yang YR, Lee CC et al. Balance outcomes after additional sitto-stand training in subjects with stroke: a randomized controlled trial. Clin Rehabil. 2010;24(6):533-42.

18. Mercer VS, Chang SH, Williams CD et al. Effects of an exercise program to increase hip abductor muscle strength and improve lateral stability following stroke: a single subject design. J Geriatr Phys Ther. 2009;32(2): 6-15.

19. Um YJ, Jang HY, Lee SM. Taping therapy simultaneously applied to the ankle and hip joint: effect on balance and gait in patients with chronic stroke. J Kor Phys Ther. 2019;31(1):49-55.

20. Park JH, Kim YM, Lee NK. The effects of repetitive sit-to-stand training with a paretic-side asymmetrical foot position on the balance of chronic stroke subjects. J Kor Phys Ther. 2015;27(3):169-73.

21. Engardt M, Grimby G. Adapted exercise important after stroke. Acute and long-term effects of different training programs. Lakartidningen. 2005;102(6):392-4.

22. Bohannon RW, Smith MB. Interrater reliability of a modified Ashworth scale of muscle spasticity. Phys Ther. 1987;67(2):206-7.

23. Butler PB, Thompson N, Major RE. Improvement in walking performance of children with cerebral palsy: preliminary results. Dev Med Child Neurol. 1992;34(7):567-76. 